\title{
Políticas de igualdad de género y sociales en España: origen, desarrollo y desmantelamiento en un contexto de crisis económica
}

\author{
Emanuela LOMBARDO \\ Universidad Complutense de Madrid \\ elombardo@cps.ucm.es \\ Margarita LEÓN \\ Universidad Autónoma de Barcelona \\ Margarita.Leon@uab.cat
}

Recibido: 23.07 .2014

Aceptado: 22.01.2015

\begin{abstract}
RESUMEN:
Este artículo hace un recorrido a través de las políticas de igualdad en España, en relación con las políticas sociales, desde su origen, desarrollo, y desmantelamiento en un contexto de crisis económica. Las políticas de igualdad de género han tenido un desarrollo espectacular en España desde principios de la democracia. Se han institucionalizado gracias a la creación de organismos de igualdad en todos los niveles de gobierno, han diversificado sus instrumentos incluyendo planes, leyes y unidades de género, y han generado unos avances que desde 2004 hasta 2008 indicaban cierta consolidación. Sin embargo, el retroceso provocado por las políticas de 'austeridad' adoptadas a partir de 2008 en respuesta a la crisis económica ha mostrado una falta de priorización de las políticas de igualdad cuando estas entran en conflicto con otras prioridades económicas. En este contexto, a pesar de la movilización de la sociedad civil, las políticas de igualdad en España tienen por delante un camino tremendamente incierto.
\end{abstract}

Palabras clave: Políticas de igualdad de género; Políticas sociales; España; Crisis económica; Políticas de austeridad.

\section{Gender Equality Policies and Social Policies in Spain: Origin, Development and Dismantlement in a context of economic crisis.}

\begin{abstract}
This article overviews gender equality policies in Spain, and their relation with social policies, from their origins, through their development and dismantlement in the economic crisis context. Gender equality policies have greatly developed in Spain from the beginnings of democracy. They have been institutionalized thanks to the creation of gender equality machinery at all governmental levels; they have diversified their instruments including plans, laws and gender units; and their progress from 2004 to 2008 indicated certain consolidation. However, the backlash provoked by austerity policies adopted
\end{abstract}


in response to the economic crisis since 2008 have shown a lack of prioritization of equality policies when there are conflicting economic priorities. In this context, despite civil society's mobilizations, the future of gender equality policies in Spain looks extremely uncertain.

Keywords: Gender Equality Policies; Social Policies; Spain; Economic Crisis; Austerity Policies.

\section{INTRODUCCIÓN}

La igualdad de género y las políticas sociales han tenido un desarrollo espectacular en España desde principios de la democracia, con desarrollos en los derechos civiles, políticos y sociales de las mujeres. En algo más de tres décadas hemos asistido a una progresiva institucionalización de organismos de igualdad en los distintos niveles de gobierno. Los instrumentos de las políticas de igualdad se han diversificado, desde planes, a leyes, y unidades de género, generando unos avances en políticas públicas contra la desigualdad de género que en los años desde 2004 hasta 2008 parecían mostrar una cierta solidez. El impulso a las políticas de igualdad y sociales ha venido de la mano de una visión política socialdemócrata y de un importante proceso de Europeización tanto a nivel cognitivo como en el acervo de 'maneras de hacer' desconocidas en nuestro país (Moreno y Serrano 2011). Sin embargo, las estrategias de control del déficit público adoptadas en Europa y en España a partir del 2008 como respuesta a la crisis económica y en el marco de un proyecto político neoliberal, han puesto freno a lo que hasta el momento era una clara trayectoria ascendente. El progresivo desmantelamiento de las políticas sociales y de igualdad y el retroceso para los derechos de las mujeres que esto conlleva, muestran una falta de priorización en materia de políticas sociales cuando éstas entran en conflicto con intereses de índole económica, tanto por parte de gobiernos conservadores como socialdemócratas. Exponen, además, los límites de las políticas en etapas anteriores a la crisis en relación con la escasa integración de una perspectiva transversal de género y la ausencia de un conjunto coordinado, completo y reconocible de medidas sociales. El recorrido por las políticas sociales y de igualdad en España, desde la democracia hasta el actual contexto de austeridad, nos hace concluir que estas políticas tienen por delante un camino tremendamente incierto.

En este artículo nos proponemos hacer un análisis de las políticas de igualdad de género en España, desde su origen hasta la coyuntura actual. Estudiamos las políticas de igualdad en el marco de las políticas sociales, no solamente porque son uno de los componentes de estas políticas, sino también porque las respuestas políticas a la crisis económica -en el marco de la 'austeridad'han afectado a todas las políticas sociales. Analizaremos la influencia de la Unión Europea en el desarrollo de este ámbito de política pública en nuestro país. Posteriormente nos centramos en el análisis de la crisis económica y de las 
consecuencias que tienen las medidas de ajuste presupuestario en las políticas de igualdad. Atestiguamos un claro recorrido descendiente de las políticas de igualdad en España. Su rescate requeriría la elección y construcción de otras alternativas políticas.

\section{ENFOQUES ANALÍTICOS}

Los enfoques analíticos que utilizamos en este artículo incluyen, por un lado, estudios sobre el feminismo de estado (McBride y Mazur 2010) y las políticas de igualdad en España (Valiente 2013; 2006; Bustelo 2004; Bustelo y Ortbals 2007), en el contexto de las políticas sociales del estado español (Guillén y León 2011). Por otro lado, incluyen enfoques teóricos neoinstitucionalistas aplicados a la comprensión de la crisis económica desde una perspectiva de género (Rubery 2014), y estudios sobre el impacto de la crisis económica y las políticas de austeridad sobre las políticas de igualdad (Bettio et al 2012; Karamessini y Rubery 2014; Gálvez 2013).

El primer bloque de estudios nos permite entender la creación y desarrollo de las políticas de igualdad en España a partir de la creación de organismos de igualdad impulsores de estas políticas, y la progresiva introducción de instrumentos de políticas de igualdad como los planes o las leyes de igualdad (McBride y Mazur 2010; Valiente 2013; Bustelo y Ortbals 2007; Bustelo 2014). Estos estudios nos permiten contextualizar las políticas de igualdad de género, identificar sus avances y limitaciones.

El enfoque del neoinstitucionalismo histórico nos ofrece instrumentos analíticos para seguir el desarrollo de las políticas de igualdad de género en España a partir de los legados institucionales y normativos de cada país (Thelen 1999), y para comprender la crisis económica como una 'coyuntura crítica' ('critical juncture'). Según Rubery (2014), las políticas de 'austeridad' representan una 'coyuntura crítica' para las políticas de igualdad y sociales en Europa que podría hacer retroceder los avances conseguidos en estos ámbitos en los países europeos desde el final de la segunda guerra mundial. Los cambios producidos por las políticas de austeridad en el mercado de trabajo, en los estados de bienestar y en las relaciones de género son resultado de este retroceso Rubery 2014: 31). Debido a que en cada contexto co-existen diferentes ideologías acerca de las relaciones de género que compiten entre sí (igualdad, diferencia, subordinación a los hombres), incluso cuando una ideología de igualdad de género emerge como dominante, existe aun la posibilidad de que ideologías de género más conservadoras revivan. 
La coyuntura de la crisis puede dar lugar a diferentes desarrollos institucionales. Uno de ellos es el del 'desplazamiento' de ideologías más igualitarias en beneficio de otras de carácter más conservador donde se reinterpretan los roles tradicionales de género. Esto es más difícil en contextos en los que existe una fuerte ideología de igualdad de género y una integración continuada de las mujeres en el mercado de trabajo. Otro desarrollo es el de la 'conversión' de instituciones pre-existentes, que toman otros significados y funciones con el cambio de las circunstancias, por ejemplo transformando una política antes progresista en una más opresiva para determinado grupos. Un tercer desarrollo es la 'desviación' ('drift'), que implica que las instituciones relajan el cumplimiento de determinados compromisos al cambiar las condiciones del contexto; es lo que está ocurriendo con el declinar de los compromisos europeos con la igualdad de género a la luz de prioridades económicas que se consideran más importantes (Rubery 2014; Bettio et al 2012).

Estudios sobre el impacto de las políticas de austeridad en los países europeos como el de Bettio et al (2012) y Karamessini y Rubery (2014) observan que mientras la recesión ha golpeado en mayor medida la ocupación masculina, por las características de la estructura productiva, las medidas de austeridad que recortan el gasto y los empleos públicos tienen un impacto más desfavorable para las mujeres. Estos análisis se centran, además, en las consecuencias diferenciadas de estas medidas dependiendo de las características de los regímenes de género preexistentes a la crisis, de las diferencias de género en el mercado de trabajo y en el cuidado, o del tipo de institucionalización de las políticas de igualdad. Junto a los otros estudios mencionados, estos análisis proporcionan unos instrumentos analíticos para comprender el origen, desarrollo y progresivo desmantelamiento de las políticas de igualdad en España.

\section{ORIGEN Y DESARROLLO DE LAS POLÍTICAS DE IGUALDAD DE GÉNERO Y SU RELACIÓN CON LAS POLÍTICAS SOCIALES DESDE PRINCIPIOS DE LA DEMOCRACIA (1975-2007)}

Con la restauración de la democracia en 1975, España inicia un proceso de modernización institucional y legislativa que supuso un giro de ciento ochenta grados en materia de igualdad de género y políticas sociales en relación a la situación heredada del franquismo. En realidad, cuando la Constitución de 1978 reconoce explícitamente la igualdad entre mujeres y hombres, con el consiguiente esfuerzo por eliminar legislación discriminatoria y contraria al principio de igualdad, la sociedad española ya había creado el espacio para que dichos cambios acontecieran. Desde los años sesenta, los rápidos cambios socio-demográficos dejaron obsoletas políticas pensadas bajo el prisma autoritario y patriarcal. Los 
movimientos migratorios campo-ciudad, una creciente y constante participación femenina en el mercado de trabajo y procesos inequívocos de secularización provocaron cambios en valores, actitudes y comportamientos que ya poco tenían que ver con los rígidos principios ordenantes de las políticas sociales. En este escenario social tan cambiante, las políticas de familia, y otras políticas sociales y fiscales que reforzaban el papel secundario de las mujeres como madres y esposas, se quedaron obsoletas.

La democracia representó un gran avance desde el punto de vista de los derechos civiles de las mujeres. El primer gobierno de UCD introdujo la legislación por la igualdad de salario en 1980; el divorcio por consentimiento mútuo en 1981; la igualdad de mujeres y hombres dentro del matrimonio; y la igualdad de hijos/as nacidos/as fuera de la unión matrimonial. Aunque la titularidad de las prestaciones familiares siguió en gran medida asociada al cabeza de familia, en general, las políticas sociales dirigidas a las familias dejaron de ser un campo activo de actuación, manteniendo su carácter asistencial. Algunas de estas medidas, especialmente la legalización del divorcio, fueron la punta del iceberg que terminó provocando una brecha profunda entre el entonces primer ministro Adolfo Suárez y la cúpula de la Iglesia Católica Española (El País 1981).

Con la victoria del PSOE en 1982, la agenda sobre igualdad de género adquiriría un protagonismo desconocido hasta el momento, impulsado por el incipiente proceso de europeización (León 2011: 62) y por el desarrollo del feminismo de estado (Valiente 2013; 2006; Bustelo y Ortbals 2007; Bustelo 2014). En 1983, gracias al activismo de las mujeres dentro del partido socialista y a la presión de los movimientos feministas, se crea el Instituto de la Mujer, con competencias a nivel nacional para la lucha contra la desigualdad de género. Los planes de igualdad se convierten en el principal instrumento de promoción y desarrollo de las políticas de igualdad, según el modelo de los Planes de Acción europeos (Bustelo 2004; Valiente 2006). El primer Plan de Oportunidades PIOM 1988-90 sienta las bases para analizar la discriminación y sus consecuencias; formación de profesionales y técnicas, desarrollo de estrategias y procedimientos. Es en este periodo cuando se crea un entramado administrativo alrededor de la problemática de la igualdad de género con la dotación de organismos para la igualdad en los distintos ámbitos de gobierno multinivel. Se introducen asimismo planes de acción positiva para combatir la discriminación por razón de sexo en el trabajo con un cierto grado de transversalidad entre ministerios. El Fondo Social Europeo financió también programas ocupacionales dirigidos específicamente a las mujeres. En estos años se legisla a favor de la legalización del aborto (1985), considerada como una de las grandes victorias del movimiento feminista aunque las restricciones de los tres supuestos legales para ejercer el derecho fueron también fuertemente criticadas por parte de grupos de mujeres que presionaron para la 
aprobación del derecho al aborto libre. Éstos acusaron al gobierno de González de sucumbir a las presiones de la cúpula de la iglesia católica.

En general, el impulso a la igualdad de género se benefició en estos momentos iniciales de la impronta socialdemócrata que dominaba buena parte de la escena europea. Sin embargo, en materia de políticas sociales y laborales y su impacto en la igualdad entre mujeres y varones, el balance fue mucho menos prometedor. A pesar del impulso institucional y político para la universalización de los ámbitos principales del estado de bienestar (fundamentalmente educación, salud y pensiones), y facilitar la entrada de las mujeres en el mercado laboral, su acceso estuvo en buena medida limitado por las dinámicas de la política de restructuración económica, comprometida por niveles elevados de desempleo. Ya desde principios de los años ochenta y en las sucesivas reformas del mercado laboral, la posición periférica de las mujeres dificultaría su integración en condiciones de igualdad al mercado productivo, ahondando en la segmentación y dualidad laboral. Así, a pesar de avances inequívocos en el acceso de las mujeres a derechos sociales básicos, la difícil entrada al mundo del trabajo y el diseño de un sistema de protección social que vincula prestaciones sociales y empleo fueron y son obstáculos importantes para una igualdad efectiva entre varones y mujeres, con incidencia de las variables socio-económicas, edad, nivel educativo, y origen además del género.

En la década de los noventa se consolida la institucionalización de las políticas de igualdad de género. El Segundo Plan de Oportunidades 1993-5 (PIOM 1993-5) amplía el ámbito de actuación considerando por primera vez el impacto de la 'doble carga' en las opciones profesionales de las mujeres. Fue este segundo plan el que reconoció la importancia de la conciliación entre la vida laboral y la familiar y la repercusión que las responsabilidades ejercidas en el ámbito privado tienen sobre las opciones de desarrollar una carrera laboral (Guillén et al. 2010: 127).

Por otra parte, es en esta década cuando se realiza la trasposición de importantes directivas europeas. La ley 42/1994 de 30 de Diciembre aplicó a la regulación española la directiva europea 92/85/EEC sobre la protección a las trabajadoras embarazadas y el derecho a la lactancia. Hasta ese momento, la baja por maternidad estaba incluida dentro de la contingencia de invalidez y enfermedad. El cambio más importante fue el pasar de considerar al permiso por maternidad como baja por enfermedad a aceptarla como una contingencia específica que requería una regulación propia. La reforma de 1994 amplió el periodo del permiso (de 14 semanas en 1989 a 16 en 1994) y la cuantía de la prestación (de $75 \%$ al $100 \%$ de la base a reguladora). El acceso a la prestación también mejoró al reducir el periodo mínimo de cotización de un año a 180 días y al asumir el estado el coste de las cotizaciones a la seguridad social. Estos cambios consiguieron una convergencia europea en esta materia. 
No obstante, a principios de los años noventa las medidas dirigidas a la protección a las familias en general eran claramente deficitarias. Los ecos de la política familiar franquista seguían coartando la renovación del discurso político, sobre todo a los partidos de izquierda. La distancia (temporal) con el régimen y a su vez la distancia (ideológica) con los gobiernos de Felipe González permitieron al gobierno del PP a partir de 1996 actualizar las políticas de apoyo a las familias (Salido y Moreno 2009). Siguiendo en buena medida las recomendaciones de la Estrategia Europea de Empleo, la mayoría de las medidas adoptadas estuvieron encaminadas a facilitar y proteger la participación de las mujeres, y madres jóvenes en particular, en el mercado laboral. La Ley para la conciliación entre la vida familiar y la laboral aprobada en 1999 reconoce abiertamente la necesidad de apoyar con medidas específicas a las personas con responsabilidades de cuidado en el ámbito familiar. La ley concluyó la trasposición de la directiva europea sobre maternidad y lactancia y en parte también la Directiva Europea 96/34/CE sobre permisos parentales. Modificó el Estatuto de los Trabajadores en relación a bajas por maternidad, paternidad y cuidado de familiares en situación de dependencia. Contempló la flexibilidad en la jornada laboral para posibilitar la lactancia materna e hizo extensibles los derechos de baja por maternidad a madres adoptivas.

En la segunda legislatura del gobierno de Aznar se introduce el Plan de Apoyo Integral a las Familias (2001-2004). Las dos medidas adoptadas más señaladas fueron la desgravación fiscal de 1.200 euros anuales -con posibilidad de fraccionar los pagos en cuotas de 100 euros mensuales- por hijo/a menor de 3 años, y los subsidios a las empresas que emplearan a mujeres (RD 3/2003) (Ministerio de Empleo y Asuntos Sociales 2010). Una evidencia clara de la capacidad de los gobiernos del PP de reformular un dominio de política pública que había sido abandonado a la deriva desde la transición democrática, fue la continuidad de algunas de las medidas de apoyo a las familias adoptadas por los gobiernos del PSOE que le sucedieron, en especial las desgravaciones fiscales por hijos a cargo.

Desde mediados de los años noventa hasta el final del ciclo expansivo de la economía, la participación de las mujeres en el mercado laboral aumentó de manera espectacular (León y Migliavacca 2013; León y Pavolini 2014). Buena parte del empleo creado durante este periodo fue empleo femenino, lo cual contribuyó a reducir la diferencia con la media europea. Sin embargo, un porcentaje elevado de los nuevos empleos fueron temporales, con unos niveles muy bajos de trabajo a tiempo parcial estable lo cual de por sí limita las oportunidades genuinas de madres y padres trabajadores de conseguir una conciliación aceptable entre sus vidas laborales y las familiares.

Por otra parte, los años que precedieron inmediatamente a la crisis económica supusieron un avance muy significativo en políticas de igualdad de 
género. La formación en 2004 del primer gobierno paritario en la historia de España fue el primer gesto del recién elegido Primer Ministro, Rodríguez Zapatero, hacia el feminismo. Los organismos de igualdad se consolidaron institucionalmente: dentro del Ministerio de Empleo y Asuntos Sociales se crea en 2004 una Secretaría General de Políticas de Igualdad, dirigida por la socióloga feminista Soledad Murillo; y se establece también en 2008 por primera vez un Ministerio de Igualdad que se ocupa no solamente de igualdad de género (al incorporar la Secretaria General de Políticas de Igualdad, el Instituto de la Mujer y la Delegación Especial para la Violencia de Género) sino también de otras desigualdades (al incorporar el Instituto de le Juventud y el Consejo para la promoción de la igualdad de trato y la no discriminación de las personas por razones de origen racial y étnico (Bustelo 2009; Lombardo 2009).

La consolidación de las políticas de igualdad se hace patente en la primera legislatura de Zapatero, con la adopción de las primeras leyes estatales de igualdad, que complementan instrumentos políticos ya en uso como los planes de igualdad o las unidades de género (Bustelo 2014; Valiente 2013). La primera ley adoptada por el gobierno Zapatero en 2004 es un símbolo del compromiso que el gobierno socialista toma con la igualdad. La Ley Orgánica 1/2004 de medidas de protección integral contra la violencia de género, votada por unanimidad por todos los partidos políticos, responde a una petición del movimiento feminista español que se movilizó desde finales de los años noventa para que la lucha contra la violencia de género entrara en la agenda política de manera prioritaria. Es una ley pionera en Europa por su diagnóstico de la violencia como un problema estructural de desigualdad de género, que los poderes públicos deben de solucionar con medidas integrales (Bustelo et al 2007). Incluye medidas en una multiplicidad de ámbitos, dirigidas a la prevención, asistencia a las víctimas, y sensibilización.

La Ley para la Promoción efectiva de la igualdad entre mujeres y hombres del 2007 (Ley Orgánica 3/2007 de 22 de marzo), conocida como Ley de Igualdad, identifica tres áreas principales de intervención: empleo, mejoras en los permisos parentales, y paridad (40/60) en las listas electorales de los partidos políticos y en los comités ejecutivos de empresas. Además, fija el principio de mainstreaming o transversalidad de un enfoque de género en las políticas públicas, y establece las unidades de género como estructuras que dentro de cada ministerio se encargarán de aplicar dicho enfoque. En lo referente a los permisos por nacimiento de hijo/a, la nueva ley mejora las prestaciones existentes, especialmente para aquéllas mujeres en una situación de mayor vulnerabilidad. La Ley concede el derecho a la baja por maternidad a mujeres menores de 21 años, mujeres desempleadas y aquéllas que no reúnan el mínimo tiempo necesario para acceder a la prestación. Por otra parte, completando la transposición de la directiva europea 96/34, reconoce un permiso exclusivo de paternidad de 13 días remunerados al 100\%, que iría aumentando 
progresivamente hasta alcanzar los cuatro meses de baja en el 2013. Además, se planificó una ampliación de cuatro semanas en el derecho de permiso de paternidad con la ley 9/2009, que tenía que entrar en vigor en 2011. Sin embargo, la irrupción de la crisis impidió como veremos más adelante, la ejecución de muchas de las medidas recogidas en la ley del 2007, incluyendo la expansión del permiso por paternidad.

En el 2005 se aprueba la equiparación de derechos y obligaciones a través del reconocimiento de plenos derechos a las parejas de hecho y la legalización del matrimonio homosexual (Ley 13/2005). Se reconoce también el derecho de adopción y paternidad a las parejas formadas por personas del mismo sexo. La ley sobre matrimonio homosexual fue criticada duramente por la Iglesia por interpretar que suponía un ataque a la institución familiar. El Partido Popular interpuso una apelación en el Tribunal Constitucional por entender que la nueva ley modificaba "la concepción secular, constitucional y legal del matrimonio como unión de un hombre y una mujer". En 2012 el pleno del Tribunal Constitucional avaló la constitucionalidad de la ley. Tras conocer la sentencia, el entonces Ministro de Justica Alberto Gallardón, declaró la no intención de modificar la nueva norma a pesar de contar con una mayoría absoluta que le habría dado la posibilidad. Se hacía así eco el gobierno de un apoyo social mayoritario hacia el matrimonio homosexual ${ }^{1}$.

Las políticas de atención a la infancia y a las personas dependientes aunque no son, en sentido estricto, políticas de igualdad de género, sí que inciden de manera particular en la igualdad entre mujeres y hombres, por una parte porque la responsabilidad del cuidado recae abrumadoramente sobre las mujeres y por otra, porque esta realidad incide directamente sobre el acceso de las mujeres al empleo (Delgado 2007).

En España existe una gran cobertura de servicios de cuidado para niñas y niños de 3 años, pero es menor la de 0-2. La educación infantil pre-escolar se incluyó en el sistema nacional de educación en 1990, a través de la Ley de Ordenación General del Sistema Educativo (LOGSE). Anteriormente, la educación de niños y niñas por debajo de la edad escolar obligatoria era competencia exclusiva de los servicios sociales de cada Comunidad Autónoma. El nuevo marco legal de los noventa fue un paso muy importante en cuanto a la expansión del la educación

\footnotetext{
${ }^{1}$ Según el Barómetro del Centro de Estudios Sociológicos (CIS 2004), pocos meses antes de la aprobación de la ley, casi la mitad (48.2\%) de los encuestados estaba de acuerdo o muy de acuerdo con la afirmación de que 'las parejas homosexuales deben de tener el mismo derecho a adoptar niños como las parejas heterosexuales (CIS 2004: 9).
} 
infantil en el tramo 3 a 6 , que alcanza niveles de prácticamente $100 \%$ de escolarización para todo el territorio español, cumpliendo así con los objetivos marcados a nivel Europeo (León 2007). Sin embargo, el principal reto para España desde el punto de vista tanto del empleo de las madres como de la igualdad de oportunidades de niños y niñas sigue estando en el tramo de 0 a 3. Aunque se produjo un aumento en la oferta en los años anteriores a la crisis con un compromiso político hacia la inversión en servicios a la infancia, a través del programa gubernamental de impulso a la educación infantil Educa3 en 2008, los niveles de cobertura siguen siendo todavía muy escasos con pocos criterios que establezcan la calidad del servicio y con un claro dominio del sector privado (Ibáñez y León 2014). Por otra parte, al formar parte del ámbito municipal de los servicios sociales y no del ámbito educativo, como ocurre con el tramo de 3 a 6 , su desarrollo es muy desigual entre las distintas Comunidades Autónomas. En 2012 sin embargo, el Ministro de Educación anunciaba la paralización del programa Educa3 junto con otras medidas de contención de gasto en el sector educativo, bajo la justificación que la etapa 0-3 tiene un carácter fundamentalmente asistencial ( $E l$ País 04/12/2012).

En relación con el cuidado de personas dependientes, se aprueba en 2006 la Ley 39/2006 conocida como Ley de Dependencia, que ha sido considerada un necesario cuarto pilar del estado de bienestar español al asignar al estado una parte de la responsabilidad en la provisión del cuidado para personas mayores y dependientes (Guillén y León 2011). Sin embargo, la ley ha sido también criticada por activistas y académicas feministas por diferentes razones (Baeza y PérezOrozco 2007). Entre ellas, la infravaloración del trabajo de cuidado que mantiene la falta de atención por las intersecciones de género, clase social y origen étnico que implica la prevalencia de mujeres inmigrantes como cuidadoras, y el hecho de que la familia, y especialmente las mujeres (el $83 \%$ de las cuidadoras no profesionales son mujeres) siguen llevando la carga principal del cuidado, teniendo el estado un papel meramente subsidiario (Peterson 2011).

A pesar de las limitaciones, los avances en las políticas de igualdad de género españolas en la década del 2000 son indudables. Dichos avances, junto con la constante incorporación de las mujeres en el mercado de trabajo, se reflejan en el tipo de 'regimen de género' que se encuentra en España en la etapa anterior a la crisis económica (Lombardo 2014). Aplicando los análisis de Walby (2009b) al caso de España, se valora que el país se ubica en el medio de un continuum entre un régimen doméstico (con baja participación laboral y política de las mujeres) y uno público (en el que se invierte la tendencia), que durante las legislaturas de Zapatero se fue acercando cada vez más hacia este último (Lombardo y Bustelo 2012). Esta ubicación se explica por el hecho de que, a pesar de que el familismo y la división tradicional del trabajo entre mujeres y hombres siguen manteniendo una carga de trabajo no 
remunerado de cuidado bastante alta para las mujeres españolas, las tasas de empleo de las mujeres han ido aumentando desde los años ochenta, llegando a un pico de $54.7 \%$ en 2007, justo antes de la crisis económica ${ }^{2}$ (Eurostat 2012, León y Migliavacca 2013). El movimiento hacia un régimen de género público en España antes de la crisis se nota también en la estabilización de la representación política de las mujeres en los parlamentos nacionales desde 2004 alrededor del 36\%, con porcentajes más bajos en el Senado (IPU 2012). Los gobiernos paritarios de Zapatero en 2004 y 2008 también son indicadores de un movimiento hacia un régimen de género público. Este impulso paritario ha podido ejercer cierta influencia en el gobierno conservador elegido en 2011, con más mujeres que en gobiernos anteriores (Bustelo 2014).

\section{LA INFLUENCIA DE LA UNIÓN EUROPEA EN LA INTRODUCCIÓN Y DESARROLLO DE POLÍTICAS DE IGUALDAD EN ESPAÑA}

La integración de España en la Unión Europea (UE) en 1986 supuso un gran empuje para la legitimización de las políticas de igualdad y la institucionalización de los organismos de igualdad. Al ingresar en la Unión, España tuvo que adaptar su legislación nacional a la normativa europea en materia de igualdad de género, lo que produjo cambios no solamente en el contenido de las políticas sino también en los estilos de policy-making y en el discurso de actores políticos (Lombardo 2003). Aun considerando que las políticas de género españolas se han generado y desarrollado gracias a la labor de las feministas de estado tanto en el nivel Estatal como Autonómico (Bustelo y Ortbals 2007; Valiente 2006), la europeización de las políticas de igualdad españolas ha tenido consecuencias relevantes tanto para la introducción de políticas e instituciones en materia de igualdad, como para las oportunidades de estrategias políticas y discursivas de los organismos de igualdad y las asociaciones de mujeres.

La UE proporciona a las políticas de igualdad de género españolas un marco de referencia desde un punto de vista normativo, político, y social (Lombardo 2004). Si políticamente la UE contribuye al proceso de democratización de España, desde el punto de vista normativo proporciona un marco legal sobre el que introducir nueva legislación, promueve la creación y desarrollo de las organizaciones de mujeres y asegura la continuidad de las políticas de igualdad en España incluso durante épocas de gobiernos conservadores. Desde un punto de vista

\footnotetext{
${ }^{2}$ Sin embargo, las tasas de empleo de las mujeres en España han sido siempre más bajas de las de los hombres (76.2\% en 2007) (Eurostat 2012). Además, la cualidad de los empleos femeninos ha estado limitada a contratos a tiempo determinado para breves periodos, mientras que una alta proporción del trabajo de las mujeres está realizado por las mujeres en la economía formal y no contabilizada en las estadísticas oficiales (Peterson 2011).
} 
social, los intercambios transnacionales, fomentados por los programas comunitarios y sus incentivos económicos, impulsan la movilización transnacional de las mujeres en Europa y los contactos entre asociaciones de mujeres. Estos contactos estimulan el conocimiento y la difusión de normas y valores acerca de la igualdad, dado que el intercambio de informaciones y buenas prácticas permite mejorar las estrategias políticas y los contenidos de las reivindicaciones.

El 'impacto domestico de Europa' (Börzel y Risse 2003) ha sido determinante en España sobre todo en materia de antidiscriminación por otras desigualdades además del género. Las directivas comunitarias sobre antidiscriminación por origen étnico o racial (2000/43/CE) y por edad, orientación sexual, discapacidad, religión o creencia (2000/78/CE) han impulsado en España desarrollos tanto institucionales como normativos. La transposición de la directiva comunitaria 2000/43/CE dio lugar a la creación en el 2003 del Consejo para la promoción de la igualdad de trato y la no discriminación de las personas por razones de origen racial y étnico (Ley 62/2003), (Bustelo 2009; Lombardo y Bustelo 2012). Sin embargo, el Consejo no se establece formalmente hasta 2007 (RD 1262/2007), empezando a trabajar en 2009 (RD 1044/2009) (Alonso et al $2012)^{3}$.

Precisamente con la crisis financiera y económica que golpea Europa desde 2008, la percepción de la UE como promotor de democracia e igualdad que había existido en España hasta esta fecha cambia, especialmente a partir de 2010 (Lombardo 2013). Incluso el Parlamento Europeo, que suele ser la mejor evaluada de las instituciones europeas, ha pasado de recibir un $62 \%$ de confianza por parte de la opinión pública española en 2008, a recibir tan sólo un 25\% de confianza en 2012 (Eurobarómetro 2008 y 2012). Cada vez más se asocia a la UE con normas y discursos que apoyan el proyecto neoliberal de reformas y austeridad que ha afectado y afecta de manera muy concreta a las políticas sociales y de igualdad.

En el contexto de la crisis económica, la europeización se percibe cada vez más como una 'intrusión' de la Unión Europea en la soberanía nacional. La presión de la UE sobre los procesos de reforma en nuestro país aumenta considerablemente cuando el gobierno español se ve obligado a solicitar asistencia financiera externa para recapitalizar y reestructurar el sector bancario español (Pavolini et al. 2014). Las condiciones del préstamo que el gobierno español recibe de la UE mediante el Fondo Europeo de Estabilidad Financiera son duras en cuanto a las reformas

3 Sin embargo, en el 2007 la Comisión Europea avisa a España de la incorrecta transposición de la directiva 2000/43/CE. En Mayo 2011 se presenta la propuesta de ley de Igualdad de Trato -que tendría que regular las otras desigualdades presentes en la directiva 2000/78/CE- y que a día de hoy no ha sido aprobada. 
políticas que las instituciones europeas piden al gobierno (reforma del mercado laboral, consolidación fiscal, privatizaciones) y al nivel de vigilancia al que el Banco Central Europeo y la Comisión Europea someten las autoridades españolas. Los recortes de gasto público efectuados por el gobierno español en el marco de las llamadas 'políticas de austeridad' tienen consecuencias negativas para la igualdad de género y las políticas de igualdad (González y Segales 2014).

\section{CRISIS ECONÓMICA, POLÍTICAS DE 'AUSTERIDAD', Y DESMANTELAMIENTO DE LAS POLÍTICAS DE IGUALDAD DE GÉNERO EN ESPAÑA}

La crisis económica que sacude Europa a partir de 2008 y que deja sus rastros más evidentes en España a partir de 2010 es un momento de 'coyuntura crítica' para las políticas de igualdad en España. Lo que empieza como crisis financiera se transforma en crisis de la deuda pública cuando los estados, esforzándose por estabilizar a los mercados, cargan con la deuda del sector financiero rescatando a los bancos fallidos (Rubery 2014; Walby 2009a), endeudándose cada vez más. La interpretación a nivel europeo de la deuda pública como causa principal de la crisis económica-que transforma en causa lo que es en realidad un efecto de la crisis financiera y en el caso español también del estallido de la burbuja inmobiliaria- nos permite comprender las razones del tipo de respuestas políticas a la crisis adoptadas por la Unión Europea y el gobierno español, justo con otros estados de la Eurozona (Busch et al 2013).

La respuesta política a la crisis económica ha sido la llamada 'austeridad', que consiste en una serie de medidas que regulan la política económica provocando unos ajustes estructurales gracias a la reducción de los salarios y del gasto público (Addabbo, Rodríguez, y Gálvez 2013: 5). Aunque Gálvez (2013) nos recuerda que dichas medidas no son otra cosa que las 'viejas políticas deflacionistas' adoptadas en los años ochenta por los gobiernos conservadores de Thatcher y Reagan en respuesta a la crisis de la economía capitalista de los setenta, el énfasis discursivo en la 'austeridad' introduce un componente moralizador nuevo que en buena medida responsabiliza y culpabiliza a la ciudadanía de la situación facilitando a los gobiernos la imposición de recortes del gasto público y aumentando el control social de la población (Addabbo, Rodríguez y Gálvez 2013: 5; Gálvez 2013).

El retroceso en políticas de igualdad debido a las políticas de 'austeridad' y a la priorización de objetivos económicos frente a los sociales es patente en el nivel europeo. El informe de la red de expertas en igualdad de género de la Comisión 
Europea de muestra que en la Unión Europea "la 'urgencia' de una respuesta a la crisis parece haber empujado al mainstreaming de género más hacia abajo en la lista de prioridades" (Bettio et al 2012: 97-98). El Plan Europeo de Recuperación Económica no menciona ni el 'género', ni la 'igualdad', ni las 'mujeres', a pesar del impacto de género diferenciado que la crisis tiene en mujeres y hombres (Bettio et al 2012; Gálvez y Torres 2010). Las Recomendaciones del Consejo Europeo para España del 2011 y 2012 para cumplir con los objetivos comunitarios acordados, incluyen medidas de consolidación fiscal y control del gasto público (por ejemplo la reforma laboral y del sistema de pensiones, las subidas de impuestos, recortes en la provisión de los servicios públicos y mayor flexibilidad para empresas). En los programas de reforma nacionales y las Recomendaciones del Consejo a España en el 2011 y 2012, el mainstreaming de género no se llevó a cabo ni en el diseño de las reformas, ni en la ejecución de las medidas de austeridad (Lombardo 2013; Bettio et al 2012; Villa y Smith 2011). Esta falta de transversalidad de género en las recomendaciones de la UE a los estados miembros para cumplir los objetivos marcados por la Europa 2020 a través de los Planes Naciones de Reforma y Estabilidad, y en la interacción con las prioridades del gobierno español, ha afectado negativamente a las políticas de igualdad de género.

A partir de 2010, tanto las políticas de igualdad como sus organismos correspondientes, sufrirían de forma directa los efectos de las políticas de 'austeridad' . Las dos olas de medidas de 'austeridad' de 2010-2011 y de 20112013 han determinado una pérdida de rango institucional o incluso desmantelamiento en las instituciones de igualdad tanto en el Estado como en las Comunidades Autónomas (González y Segales 2014). El propio gobierno de Zapatero, después de una primera legislatura de progresos sustantivos en políticas de igualdad de género y paridad democrática, suprimió en 2010 el Ministerio de Igualdad, recién creado en 2008, como una de las primeras medidas de reordenación ministerial dirigida al ahorro del gasto administrativo. La institución pasó a convertirse en un organismo de rango menor (Secretaria de Estado) ubicada en el seno del Ministerio de Salud, Política Social e Igualdad. A nivel autonómico, bajadas de rango o eliminación de las instituciones de igualdad tuvieron lugar en Galicia, Murcia y Madrid (Alonso 2012). Estos cambios implican un deterioro significativo de las instituciones por la igualdad que podría afectar su capacidad de acción política.

A pesar de que los programas de inclusión social y de igualdad de género adoptados por el gobierno socialdemócrata en 2004-2011 protegieron a las mujeres en los primeros años de la crisis económica según Lahey y de Villota (2013), las políticas de contención de gasto implementadas en el último tramo del mandato del gobierno del PSOE ensombrecieron en buena medida el espíritu progresista y de compromiso por la igualdad.. Zapatero fue acusado, incluso por personas de su 
propio gabinete, de comprometer gasto social cuando el huracán de la recesión económica global y el pinchazo de la burbuja inmobiliaria ya habían llegado para quedarse.

Con el cambio de gobierno en el 2011, las reducciones presupuestarias inducidas por las medidas de consolidación fiscal conllevan el riesgo de pérdida de visibilidad y reconocimiento de las políticas de igualdad o la disminución de las partidas presupuestarias. Como afirman Bettio et al. (2012: 113,117): "el caso español hace sonar campanas de alarma sobre las repercusiones de la crisis en la maquinaria de igualdad de género (...) '. La reestructuración de los ministerios se lleva a cabo de nuevo bajo el gobierno conservador de Rajoy. En esta ocasión, el Instituto de la Mujer pasa ahora a depender del Ministerio de Salud, Servicios sociales e Igualdad ${ }^{4}$. En la nueva estructura ministerial lo que antes era la Secretaría de Estado por la Igualdad se convierte en una Secretaría de Estado con competencias de prevención y eliminación de discriminación amplias, desde exclusión social a familia, infancia, dependencia, discapacidad e igualdad. El Instituto de la Mujer deja de ser una Secretaría de Estado para depender ahora de una Dirección General por la Igualdad de Oportunidades (situada dentro de la Secretaria de Servicios sociales e Igualdad), lo que supone un rango institucional menor. La justificación del gobierno por la reestructuración institucional tanto del 2010 como del 2011 ha sido la del ahorro en los gastos administrativos -bajo los lemas de 'racionalizar' y 'simplificar'-. Estos cambios institucionales sin duda representan una tendencia contraria al camino de consolidación de las políticas de igualdad en España.

El impacto de las políticas de austeridad adoptadas por los gobiernos españoles en respuesta a la crisis económica desde el año 2009 se hace notar también en los recursos dedicados a las políticas de igualdad de género. Paleo y Alonso (en este número) comparan la evolución de los presupuestos destinados a las políticas de igualdad tanto a nivel estatal como autonómico durante el periodo anterior a la crisis económica, desde 2002 hasta 2008, y en la etapa de crisis desde 2009 hasta $2013^{5}$. Las autoras observan que mientras durante el periodo 2002-2008 en el $90 \%$ de los casos analizados el presupuesto dedicado a las políticas de igualdad de género aumentó o se mantuvo contante, en el periodo de crisis económica tan sólo en un $25,6 \%$ de los casos este presupuesto aumentó o se mantuvo constante, y sin embargo en un $74,4 \%$ de casos el presupuesto para políticas de igualdad disminuyó. Además, Paleo y Alonso (en este número) afirman

\footnotetext{
${ }^{4}$ Véanse RD 200/2012 de 23 de Enero de 2012, RD 1823/2011 de 21 de Diciembre de 2011, y RD 1887/2011 de 30 de Diciembre de 2011.

${ }_{5}^{5}$ Los Presupuestos 2002-2013 se analizan utilizando como fuentes el Boletín Oficial del Estado y los Boletines Oficiales Autonómicos.
} 
que al analizar las variaciones totales que se producen en los presupuestos del Estado y de las Comunidades Autónomas en los dos periodos antes y durante la crisis se puede observar que en el periodo 2002-2008 en todos los casos analizados el presupuesto destinado a las políticas de género aumenta considerablemente, llegando a $57,2 \%$ a nivel estatal, mientras que en el periodo 2009-2013 el presupuesto disminuye en todos los niveles de gobierno (llegando a $-34,1 \%$ para la Administración Central del Estado), con la excepción de Andalucía donde el presupuesto destinado a las políticas de género aumenta un 15,5\%. La justificación por los recortes en materia de políticas de igualdad ofrecida por el gobierno conservador es la de la crisis económica y de la mala administración del gobierno anterior $^{6}$.

Las medidas de austeridad tienen una influencia negativa en la normativa propuesta en materia de política de igualdad y en los recortes a políticas concretas. La propuesta de una ley de igualdad de trato y no discriminación por múltiples desigualdades, presentada en enero de 2011 para completar la trasposición de la normativa comunitaria en materia de igualdad hasta la fecha se ha quedado fuera de una agenda política centrada en las medidas de austeridad (Lombardo 2013). De manera similar, la extensión del permiso de paternidad de dos a cuatro semanas a conseguir en 2011, propuesta por Zapatero en la ley 9/2009, ha quedado estancada con el argumento de la crisis económica (Bustelo 2014). Los derechos del trabajo y la ley de Dependencia también se han visto recortados de diferentes maneras, en perjuicio de la igualdad. La posibilidad de disfrutar de los derechos a conciliar el trabajo con la vida familiar se ha visto reducida por la Ley 3/2012 del 6 de Julio de Reforma Laboral. Como argumenta Ballester-Pastor (2012), la reforma laboral introduce la posibilidad de horas extraordinarias para quien trabaja a tiempo parcial (que antes estaba prohibida), poniendo a estas personas -en su mayoría mujeresaun más a disposición de quien las contrata. Además limita, entre otras cosas, la posibilidad de pedir reducción del tiempo de trabajo por razones de conciliación por periodos más largos. Estas razones llevan a Ballester-Pastor (2012: 29) a concluir que 'La reducción de los derechos parentales que ha tenido lugar con la reforma laboral española de 2012 podría tener el efecto de expulsar del mercado de trabajo aquellas personas que no consiguen compatibilizar sus varias responsabilidades'. Los recortes, a partir del 2010, han tocado también la Ley Dependencia, reduciendo el apoyo económico estatal para cuidadoras de familiares dependientes y eliminando la protección social para cuidadoras no profesionales (González y Segales 2014), y así haciendo aún más difícil la implementación de una ley para la cual ya en origen no se había asegurado una línea clara de financiación (Valiente 2013). Además, las organizaciones feministas pertenecientes a la Plataforma

${ }^{6}$ El País 24/11/2012. 
'Impacto de Género ya!' denuncian en su análisis de los presupuestos generales del Estado para el 2015 que 'pese a ser mujeres el 66\% de las personas dependientes y el $83 \%$ de sus cuidadoras, el Informe Oficial de Impacto de Género, como en años anteriores, no analiza el programa presupuestario ni su impacto en el reparto del cuidado, ${ }^{7}$.Los programas de austeridad también han alcanzado a políticas contra la violencia de género. En el presupuesto de 2015 la partida destinada a las políticas en contra de la violencia de género se recortó en un $22.5 \%$ en comparación con el presupuesto del año 2008, continuando la tendencia decreciente desde el inicio de la crisis $^{8}$. Sin embargo, comparado con otras políticas de igualdad, en el caso de la violencia de género, la capacidad de encontrar consensos entre partidos de distinto signo político (todos los partidos políticos apoyaron la aprobación de la Ley Integral contra la violencia de género en el 2004), el activismo de los movimientos feministas y sus alianzas con las feministas de estado han permitido a las políticas contra la violencia de género una cierta continuidad incluso en momentos de fuertes ajustes y reformas a partir de 2011.

La manera de enmarcar las políticas de igualdad también cambia en el periodo de la crisis en comparación con la etapa anterior. Durante las legislaturas Zapatero 2004-2011 el discurso político sobre la desigualdad de género tiende a considerar esta última como un problema causado por unas estructuras sociales desiguales que las autoridades públicas tienen que solucionar promoviendo políticas activas de igualdad (Bustelo y Lombardo 2012). La violencia de género se considera en los discursos políticos como un problema público que tiene origen en un sistema patriarcal de dominación de las mujeres por parte de los varones. En las políticas de cuidados aparecen discursos acerca de la corresponsabilidad del cuidado entre mujeres y varones, aunque la tendencia es a enmarcar el problema como conciliación de trabajo y vida familiar por parte de las mujeres (Peterson 2011). En opinión de Bustelo (2014), estos avances en el discurso han calado en la sociedad española de tal manera que sería difícil su desaparición. Aunque puedan desaparecen del discurso oficial del gobierno, se mantendrían -según la autoragracias a la labor del personal de la administración pública que trabaja en las instituciones de igualdad y en la implementación de las políticas, así como gracias a las voces del feminismo del movimiento y de la academia.

Sin embargo, los avances en la definición del problema de la desigualdad de género están puestos en riesgo en la etapa de crisis no solamente debido a la ya mencionada subordinación de la igualdad a los objetivos de austeridad, sino también a una agenda en materia de igualdad marcada por un partido de ideología conservadora. El lenguaje oficial del gobierno de 2011 habla más de 'violencia

\footnotetext{
${ }^{7}$ Véase http://impactodegeneroya.blogia.com/

${ }^{8}$ Véase http://impactodegeneroya.blogia.com/
} 
doméstica' que de 'violencia de género', poniendo así el énfasis en el lugar en el que ocurriría violencia más que en las causas estructurales o en los actores que realizan la violencia (Bustelo et al 2007; Krizsan et al 2007). La reforma del aborto que el gobierno del partido popular propuso en el Anteproyecto de Ley Orgánica de protección de la vida del concebido y derechos de la mujer embarazada tenía el objetivo de restringir este derecho, retrocediendo incluso hasta la etapa anterior a la ley del aborto de 1985. En este sentido la idea de la maternidad quedaba enmarcada dentro de un enfoque pro-vida que cuestiona la autonomía y los derechos de las mujeres (Alonso 2013). Leyes que regulan la protección de la maternidad se han aprobado en seis Comunidades Autónomas gobernadas por el Partido Popular. El cambio en la manera de situar el problema de la desigualdad de género es evidente en comparación con los marcos de la etapa progresista de 2004-2011. Estas leyes, argumentan Paleo y Alonso (en este número), se centran en el tradicional papel de las mujeres como madres, limitan su autonomía en relación con el embarazo, ofreciendo ayudas a madres en situaciones de vulnerabilidad exclusivamente si quieren seguir adelante con el embarazo (llegando en determinados casos a mencionar explícitamente los derechos del no nacido), y sin pretensión aparente de transformar los roles de género. El gobierno cedió ante la fuerte presión social, en buena parte ejercida por movimientos feministas, y finalmente decidió retirar el polémico anteproyecto de ley forzando la dimisión de su propulsor, el entonces Ministro de Justicia Alberto Ruiz-Gallardón.

Según Paleo y Alonso (en este número), la ideología de los partidos políticos es un factor explicativo no solo del tipo de política que se lleva a cabo, como es el caso de las medidas de protección de la maternidad desde una perspectiva pro-vida, sino también de los recortes en el presupuesto dedicado a políticas de igualdad, con una mayor probabilidad de recortes en políticas de género cuando estén partidos de centro-derecha en el gobierno. Aunque los partidos de centro-derecha tengan un mayor alineamiento con el proyecto neoliberal y la promoción de roles de género más tradicionales, también es cierto que las políticas de austeridad se gestan en el seno de una Unión Europea que en el contexto de la crisis económica $-\mathrm{y}$ en acuerdo con los gobiernos de los países miembros- está subordinando la igualdad de género, promovida por su propia normativa, a prioridades económicas más 'urgentes' en línea con un proyecto neoliberal (Walby 2013). El gobierno español, así como otros gobiernos europeos afectados por la crisis económica, utilizan el eslogan de Thatcher de 'no hay alternativa' a los recortes neoliberales, para legitimar las políticas de austeridad (Bonoli 2012 en Pavolini et al 2014).

Si la respuesta de la política institucional a la crisis económica está promoviendo un desmantelamiento de las políticas sociales y de igualdad, en el marco de un proyecto neoliberal, una parte de la sociedad civil española está 
políticamente activa en la defensa de los servicios públicos y los derechos sociales. La movilización ciudadana que arranca con el movimiento $15 \mathrm{M}$ o de los Indignados, y seguida por las asambleas de los barrios, la plataforma contra los desahucios (la PAH), y las mareas ciudadanas de distintos colores en defensa de la sanidad y la educación pública, así como la igualdad, es crítica con los recortes de los servicios públicos en el nombre de la crisis y denuncia la degradación de la calidad de la democracia y de la justicia social en general (Cruells e Ibarra 2013). En materia de igualdad, las movilizaciones feministas más recientes se han centrado sobre todo en la defensa del derecho de aborto. También ha habido movilizaciones para defender las políticas y recursos públicos en contra de la violencia de género. En 2011, cuando el gobierno del PP de Castilla-La Mancha anunció el 41\% de recortes en centros de acogida para mujeres maltratadas, la presión de peticiones y movilizaciones masivas de mujeres paró el plan del gobierno y consiguió salvar los centros (Lombardo 2013). La atención a las mujeres víctimas de violencia, sin embargo, está amenazada por la reforma de la ley del régimen local adoptada por el gobierno de Rajoy en 2013 como parte del plan de contención del gasto público que elimina la competencia local en políticas de igualdad.

\section{CONCLUSIONES}

Desde la restauración de la democracia española, hemos sido testigos de avances relevantes en el ámbito de la igualdad efectiva de oportunidades, la introducción de legislación no discriminatoria y de igualdad de género junto con cambios de adaptación institucional que han forzado la "prescripción" de principios patriarcales que relegaban a las mujeres a un rol subordinado. Las políticas de igualdad se han institucionalizado y desarrollado en los diferentes niveles gubernamentales, llegando a consolidarse en la primera década del 2000, con avances considerables en materia de políticas contra la violencia de género o la igualdad en el empleo. Las dos legislaturas de Zapatero, de hecho, contribuyeron de manera significativa a la convergencia europea en leyes y políticas por la igualdad.

Sin embargo, a pesar de que el principio del mainstreaming o transversalidad de género se haya incorporado en la normativa española con la ley $3 / 2007$, ha faltado en España una actuación decidida y explícita a favor de la igualdad por parte de otras políticas públicas que aunque no podamos catalogar como de igualdad en la excesiva compartimentación de las políticas públicas, sí que en cambio afecta de manera directa a las posibilidades reales de una igualdad efectiva. Esto ha implicado, por ejemplo, que el fuerte incremento de la participación laboral de las mujeres españolas haya tenido lugar sin que se produjesen cambios sustantivos en la provisión del cuidado y las prestaciones de apoyo a las familias, agudizando la "tradicional" situación de sobrecarga de las mujeres que ha caracterizado el sistema de bienestar en 
nuestro país. En resumidas cuentas, a pesar de los avances en políticas de igualdad de género, ha faltado coherencia en el desarrollo de las políticas sociales. Más que de una política social articulada con unos fines concretos y transversales, se ha tratado de un conjunto de medidas dispares, orientadas principalmente por el impulso europeo a favor de la empleabilidad de las mujeres y, en la última etapa, por una apuesta decidida por la igualdad de género que, no obstante, no ha sido capaz de darle prioridad frente a otros objetivos.

La falta de priorización de la igualdad de género y de integración de un enfoque de género en todas las políticas públicas se ha hecho patente en la 'coyuntura crítica' que la crisis económica ha supuesto. Las medidas de 'austeridad' adoptadas por parte de gobiernos europeos de corte tanto conservador como socialdemócrata han desplazado la agenda social en beneficio del equilibrio de los parámetros macro-económicos. La perspectiva desarrollada en este artículo de enmarcar el análisis de las políticas de igualdad en el contexto más general de las políticas sociales nos ha permitido captar como las políticas de 'austeridad' hayan afectado no solamente a las políticas de igualdad, sino a todas las políticas sociales. Esto ocurre en el marco de un proyecto neoliberal que promueve explícita o implícitamente olas de desregulaciones y privatizaciones, así como, en el gobierno Rajoy, apostando por unas políticas de igualdad más restrictivas de los derechos de las mujeres.

Las medidas de austeridad no han solucionado el problema de la crisis económica en España sino que han empeorado tanto las condiciones de vida de mujeres y varones como las políticas de igualdad (González y Segales 2014). Siguiendo por este camino, y si la Unión Europea no vuelve a renovar más decididamente su compromiso con la igualdad y por la integración de un enfoque de género en las medidas anti-crisis en todos los países miembros, el desmantelamiento de políticas específicas puede llevar a retrocesos importantes en igualdad.

Sin embargo, la 'coyuntura crítica' implica la posibilidad de optar por caminos diferentes. Según Walby (2013), el proyecto neoliberal es una de las posibles alternativas políticas de elección como respuesta política a la crisis económica, opuesta a la alternativa social-demócrata. Ser conscientes de que puede haber más alternativas - como las que vislumbran las movilizaciones de la sociedad civil española- es una llave de lectura importante para entender las razones del retroceso. 


\section{BIBLIOGRAFÍA}

ADDABBO, Tindara, RODRÍGUEZ-MODROÑO, Paula, y GÁLVEZ-MUÑOZ, Lina (2013): "Gender and the Great Recession: Changes in labour supply in Spain", paper presented a la 54 Conferencia Anual Alma Mater Studiorum, Universidad de Bologna 24-26 October 2013.

BAEZA, Paula y OROZCO, Amaia (2007): "Notas críticas a la nueva 'Ley de Dependencia'. Éxodo, 87(17-23).

BETTIO, Francesca et al. (2012): The impact of the economic crisis on the situation of women and men and on gender equality policies. Brussels: European Commission.

BUSCH, Klaus et al (2013): Euro crisis, austerity policy and the European social model. Friedrich Ebert Stiftung, http://library.fes.de/pdf-files/id/ipa/09656.pdf

BUSTELO, María (2014): "Three Decades of State Feminism and Gender Equality Policies in Multi-Governed Spain”. Sex Roles, Advanced online publication 15 de junio de 2014, DOI 10.1007/s11199-014-0381-9

BUSTELO, Maria (2009): "Spain: Intersectionality Faces the Strong Gender Norm". International Feminist Journal of Politics, 11(4): 530-46.

BUSTELO, Maria y Candice Ortbals (2007): "The Evolution of Spanish State Feminism. A Fragmented Landscape" En Joyce Outshoorn y Johanna Kantola (eds). Changing State Feminism, Basingstoke: Palgrave, 201-223.

BUSTELO, Maria y LOMBARDO, Emanuela (2012): "Understanding and assessing quality in gender violence policies in Italy and Spain", ponencia presentada en ECPR Joint Sessions, Antwerp 10-15 Abril 2012.

BUSTELO, Maria, LÓPEZ, Silvia y PLATERO, Raquel (2007): "La representación de la violencia contra las mujeres como un asunto de género y un problema público en España" En Maria Bustelo y Emanuela Lombardo (eds.) Políticas de igualdad en España y en Europa. Madrid: Cátedra, pp 67-96.

CRUELLS, Marta y YBARRA, Pedro coords. (2013): La democracia del futuro. Del $15 \mathrm{M}$ a la emergencia de una sociedad civil viva. Barcelona: Icaria.

DELGADO, Margarita (2007): Encuesta de Fecundidad, Familia y Valores 2006, Colección Opiniones y Actitudes, Vol. 59. Madrid: CIS.

DELGADO, M.; ZAMORA, F.; BARRIOS, L.; CÁMARA, N.; ALBERDI, I.; y DE ROSE, A. (2009): Fecundidad y trayectoria laboral de las mujeres en España. Madrid: Instituto de la Mujer.

GÁLVEZ, Lina (2013): 'Una lectura feminista del austericidio'. Revista de Economía Crítica, ${ }^{0} 15$, primer semestre 2013, pp 80-110. http://revistaeconomiacritica.org/sites/default/files/revistas/n15/Crisis02 linagalvez.pdf

GONZÁLEZ, Elvira y SEGALES, Marcelo (2014): Women, gender equality and the economic crisis in Spain. Karamessini, M. y J. Rubery eds. Women and Austerity. The Economic Crisis and the Future for Gender Equality 228-247. London: Routledge. 
GUILLÉN, Ana M. y LEÓN, Margarita (2011) (ed.) The Spanish Welfare State in European Context. Aldershot: Ashgate.

KARAMESSINI, M. y RUBERY, J. eds. (2014): Women and Austerity. The Economic Crisis and the Future for Gender Equality, London, Routledge.

LAHEY, Kathleen A. y DE VILLOTA, Paloma (2013): "Economic Crisis, Gender Equality, and Policy Responses in Spain and Canada", Feminist Economics 19(3): 82-107.

LEÓN, Margarita (2011): “The Quest for Gender Equality” En Ana Guillén y Margarita León (eds.) The Spanish Welfare State in the European Context. Aldershot: Ashgate, 59-74.

LEÓN, Margarita y MIGLIAVACCA, M. (2012): "Italy and Spain: Still the case of familistic welfare models?" En T. Martín García (ed.) Spain and Italy: As Similar as Thought? A comparative reflection on living arrangements and family relationships. Springer.

LEÓN, Margarita y PAVOLINI, Emmanuela (2014) “'Social Investment' or back to 'Familism': The impact of the economic crisis on Family and Care Policies in Italy and Spain". South European Society and Politics, 19(3): 353-369.

LOMBARDO, Emanuela (2014): Gender mainstreaming and policy responses to the economic crisis: the "unintended consequences" of $\mathrm{EU}$ and national policymaking on Spanish gender equality policies, ponencia presentada en la Universidad de Helsinki, 6 Febrero de 2014.

LOMBARDO, Emanuela y BUSTELO, Maria (2012): "Political approaches to inequalities in Southern Europe: a comparative analysis of Italy, Portugal and Spain". Social Politics._International Studies in Gender, State and Society, 19(4): 572-595.

LOMBARDO, Emanuela (2009): Spanish policy on gender equality: relevant current legislation and policies, Briefing note for the European Parliament, DG for Internal Policies, Policy Department C: Citizens' Rights and Constitutional Affairs, PE 419.612.

LOMBARDO, Emanuela (2004): La europeización de la política española de igualdad de género. Colección Ciencia Política. Valencia: Tirant Lo Blanch.

LOMBARDO, Emanuela (2003): "La europeización de la política española de igualdad de género". Revista Española de Ciencia Política, 9: 63-80.

MCBRIDE, Dorothy y MAZUR, Amy (2010): The Politics of State Feminism. Innovation in Comparative Research. Philadelphia: Temple University Press.

MORENO Minguez, A. (2007): Familia y empleo de la mujer en los regímenes de bienestar del sur de Europa. Madrid: CIS.

PALEO, Natalia y ALONSO, Alba (2015): “¿Es únicamente una cuestión de austeridad? Crisis económica y políticas de género en España", Revista de Investigaciones Feministas, 5.

PAVOLINI, Emmanuela, LEÓN, Margarita, GUILLÉN, Ana M., y ASCOLI, Ugo (2014) "From Austerity to permanent strain? The EU and welfare state reform in Italy and Spain". Comparative European Politics. (20 Octubre 2014) | Advanced online publication doi:10.1057/cep.2014.41 
PETERSON, E. (2011): Beyond the "women-friendly" welfare state: Framing gender inequality as a policy problem in Spanish and Swedish politics of care, $\mathrm{PhD}$ thesis, Madrid Complutense University.

RUBERY, Gill (2014): From 'women and recession' to 'women and austerity': a framework for analysis. Karamessini, M. y J. Rubery eds. Women and Austerity. The Economic Crisis and the Future for Gender Equality 17-36. London: Routledge.

SALIDO, Olga (2011): 'Female employment and work-life balance policies in Spain'. En M. León y Guillén, A. (eds.) The Spanish Welfare State in the European Context Farnham: Ashgate.

SALIDO, Olga y MORENO, Luís (2009): "Familia y género" En Luís Moreno (ed.) Reformas de las Políticas del Bienestar en España. Madrid: Siglo XXI.

THELEN, Kathleen (1999): 'Historical Institutionalism in Comparative Politics', Annual Review of Political Science, 2 (1), 369-404.

VALIENTE, Celia ( 2013): “Gender equality policymaking in Spain (2008-11): Losing momentum". Bonnie N. Field, and Alfonso Botti eds., Politics and society in contemporary Spain: From Zapatero to Rajoy 179-95. New York: Palgrave Macmillan.

VALIENTE, Celia (2006): El feminismo de Estado en España: El Instituto de la Mujer (1983-2003), Valencia, Universitat de Vàlencia.

WALBY, S. (2013): 'Finance versus democracy? Theorizing finance in society', Work, employment and society DOI: 10-1177/0950017013479741.

WALBY, S. (2009a): 'Gender and the financial crisis'. Paper for UNESCO Project on 'Gender and the Financial Crisis', 9 April 2009, available at http://www.lancs.ac.uk/fass/doc library/sociology/Gender_and financial_crisis Sylvia Walby.pdf

WALBY, S. (2009b): Globalization and Inequalities. Complexity and Contested Modernities. London: Sage. 